\title{
Implicaciones teórico-metodológicas en la historia presente de la educación musical de nivel superior en México
}

Irma-Susana Carbajal-Vaca

\section{RESUMEN}

Los programas de educación musical que otorgan el grado de licenciatura en México tienen, en su mayoría, una vida aproximada de tres décadas. Con el objetivo de documentar la historia presente de los programas, se asumió que comprender los significados educativos compartidos por los miembros de cada comunidad permitiría identificar tendencias no explícitas en el currículo formal. En este documento se presenta la problematización teórico-metodológica focalizada durante esta investigación que ha asumido la responsabilidad universitaria de hacer sonar las voces del presente que quedarán en la historia para contrastarse con voces futuras.

Palabras clave: historia de la educación, educación artística, educación universitaria, historia presente, México. 


\section{Implicações teórico-metodológicas na história presente da educação musical de nível superior} no México

\section{RESUMO}

Os programas de educação musical que concedem o grau de licenciatura no México têm, em sua maioria, uma vida aproximada de três décadas. Com o objetivo de documentar a história presente dos programas, se assumiu que compreender os significados educativos compartilhados pelos membros de cada comunidade permitiria identificar tendências não explícitas no currículo formal. Neste documento se presenta a problematização teórico-metodológica focalizada durante esta pesquisa que tem assumido a responsabilidade universitária de fazer soar as vozes do presente que ficarão na história para contrastarse com vozes futuras.

Palavras chave: história da educação, educação artística, educação musical, história presente, México.

\section{Theoretical-methodological implications in the current history of higher music education in Mexico}

\section{ABSTRACT}

Most music training programs that award a bachelor's degree in Mexico have a life span of approximately three decades. In order to document the current history of the programs, the author assumed that understanding the educational meanings shared by the members of each community would allow to identify the non-explicit trends in the formal curriculum. This document presents the theoreticalmethodological problematization focused during this research, that has taken over the university responsibility to make the voices of the present resonate so that they will remain in history to be contrasted with voices of the future.

Key words: history of education, artistic education, music education, current history, Mexico. 


\section{Introducción}

Para lograr la formación integral universitaria que demandan actualmente los modelos educativos, los programas de educación musical de nivel superior han ido adoptando formas híbridas, ${ }^{1}$ a manera de conservatorios universitarios (Aguirre Lora, 2006: 101102; Carbajal Vaca, 2016b), ${ }^{2}$ que abonan tanto al desarrollo de habilidades vocales e instrumentales, como al desarrollo de habilidades propias del campo musicológico. Actualmente, incluso los programas de las instituciones que se identifican como conservatorio han incluido asignaturas de disciplinas ajenas al desarrollo de habilidades musicales, las cuales parecen desviar la atención del propósito de formación musical primigenio. Un ejemplo podría verse en el objetivo de la licenciatura que ofrece el Conservatorio Nacional de Música (CNM) en México, el cual podría ya entenderse como un modelo híbrido: "Formar profesionales de la música de alto nivel en cuatro áreas de Orientación Profesional: la Interpretación, la Investigación, la Creación y la Docencia" (CNM, 2017).

En el Primer Simposio Perspectiva Histórica del Arte, realizado en 2014 en el Instituto de Artes de la Universidad Autónoma del Estado de Hidalgo, se sostuvo que documentar la historia de las instituciones, "es una necesidad y una obligación de sus integrantes (docentes, alumnos y administrativos). Si no conocemos el camino que han seguido quienes nos antecedieron, nuestras discusiones se estancarán en la emisión de juicios parciales que resultarán injustos, improductivos y equivocados" (Carbajal Vaca, 2016a).

Se enfatizó que los modelos híbridos actuales han hecho posible la integración de profesores investigadores con formación interdisciplinaria y la implementación de nuevas tecnologías en los centros de trabajo, con lo que se ha asentado un sustrato fértil para el desarrollo de proyectos de investigación colaborativos e interinstitucionales.

En 2017, Carbajal Vaca convocó a profesores e investigadores voluntarios de distintas instituciones en el país para realizar una investigación colaborativa mediante tres tratamientos interrelacionados: uno heurístico, uno etnográfico y uno hermenéutico, para interpretar el desarrollo y las tendencias que dibujan el panorama de la educación musical de nivel superior en México en los últimos 30 años.

El trabajo se insertó en el terreno historiográfico como resultado de operaciones heurísticas, hermenéuticas y críticas, no necesariemente sucesivas, sino dispuestas en conjunto sobre una arquitectura ideada en una etapa incipiente del proyecto (Gaos, 2000: 493-494); arquitectura erigida por los pilares origen, desarrollo y tendencias en las instituciones de educación superior (IES) del país que han incursionado en la formación musical de nivel licenciatura.

El estudio se relaciona con distintas disciplinas: 1) con la historia de la educación superior en México, porque es la que enmarca el contexto sociopolítico en el que se diseñaron los programas de licenciatura en música; 2) con la sociología de la música, ya que la música es una expresión social activa y vigente que provee información sobre el comportamiento de los diferentes grupos sociales que son resultado de procesos sociohistóricos complejos, y 3) con el análisis curricular, porque los programas de educación musical de nivel superior son parte del sistema universitario formal y, aunque han sido diseñados en el influjo de las políticas públicas y tendencias socioeconómicas actuales — nacionales e internacionales - también

\footnotetext{
${ }^{1}$ Programa que sigue las metas de formación del conservatorio tradicional, pero que ha incluido asignaturas que cumplen con los objetivos universitarios.

${ }^{2}$ Este concepto fue utilizado en 1929 cuando los estudiantes de la Universidad Nacional Autónoma de México (UNAM) solicitaron la creación de una Escuela de Música Universitaria. En 1933 el plan de estudios aún no se diferenciaba del plan del Conservatorio (Aguirre Lora, 2006: 104). Este antecedente de la Facultad de Música de la UNAM es importante porque es el primer programa que se ciñó a las directrices universitarias; sin embargo también continuó con la dinámica de formación conservatoriana porque sus profesores eran egresados de los conservatorios.
} 
son producto de procesos internos que han ido configurando tradiciones, marcando identidades, delineando concepciones profesionales y generando comprensiones diversas sobre la práctica musical en general y sobre las necesidades educativas.

\section{El entramado de la tradición y los sistemas culturales en la historia presente}

La concepción heurística adoptada en este trabajo se refiere a la búsqueda de información o documentos, óptica más común aceptada por los historiadores (Matute, 1999: 9), para ser interpretados (Beuchot Puente, 1999:15) y conjeturar posibles explicaciones sobre el desarrollo y tendencias en los programas de educación musical, lo que, de acuerdo con Julio Aróstegui (2004), en la perspectiva de la historia del presente conlleva problemáticas de naturaleza pragmática, como la accesibilidad a la información y el carácter de las fuentes por influencia de las nuevas tecnologías:

la calidad y cantidad de la información disponible: la lucha contra la resistencia a la información pública, la tergiversación y ocultamiento, la inaccesibilidad. Otro problema es que la revolución tecnológica del último cuarto del siglo XX nos ha llevado a una situación absolutamente nueva en cuanto al carácter de las fuentes de la historia y la transmisión de la información, precisamente porque hemos entrado en la época del informacionalismo, de la redundancia y los "ruidos", de la información que es "desinformación" (Aróstegui, 2004: 42).

El fundamento de este estudio se sitúa en la sociología del filósofo alemán Wilhelm Dilthey (18331911), concebida en el marco de las ciencias del espíritu (Geisteswissenschaften), como una vía para estudiar el desarrollo de la sociedad como un 'todo' que no puede separarse del conocimiento de su estado actual porque éste "es resultado del anterior y, al mismo tiempo, condición del próximo. El estado actual, por lo tanto, pertenece ya, en el próximo, a la historia"
(Dilthey, 1949: 44). Sobre esta premisa se sostiene que para conocer el estado actual de los programas de formación musical de nivel superior es necesario investigar la historia del presente; un tipo de historia que en un inicio, sin advertir las implicaciones epistemológicas, se conceptuó como historia reciente.

La conformación de un marco teórico-metodológico desde la perspectiva de historia reciente no es una tarea sencilla porque son múltiples los adjetivos que sugieren este espacio temporal, pero no todos enmarcan el mismo objeto. Así se encontró el término historia vinculado a los calificativos inmediata, fluente, coetánea, viviente, próxima; además de construcciones como del pasado actual, muy contemporánea, de nuestro tiempo, del mundo actual, del tiempo presente, del pasado cercano o la era del testigo (Alonso 2007; Aguirre-Lora 2016; Soto Gamboa 2004; Franco y Levín, 2007). Estos términos sumergieron la investigación en un halo de ambigüedad que reclamaba precisiones; tarea filosófica que excedía las metas del proyecto, pero que permitió problematizar algunas características propias de los programas de licenciatura en música.

Se reconocieron algunas características como acuerdo entre los historiadores para acotar un espacio posible dentro de la historia contemporánea que puede ser considerado historia reciente, a saber: 1) la supervivencia de los protagonistas del pasado, 2) que los actores estuvieran en condiciones de brindar testimonios al historiador, 3) la existencia de una memoria social viva, 4) disponer de procesos históricos inacabados o en desarrollo para ser considerados como objeto, 5) no limitar la coetaneidad entre pasado y presente a los años recientes, 6) la detección de fenómenos no muy lejanos que hayan afectado la dinámica de las sociedades, y 7) que dichos fenómenos se relacionaran con procesos sociales traumáticos, como genocidios, terrorismo o guerras (Franco y Levín, 2007: 2; López et al., 2010: 14; Arias Gómez, 2015: 30). Esta última característica es la que identifica con mayor fuerza la perspectiva de la historia reciente y la que puso en duda la utilización del concepto 
en la investigación. Se encontró que esta categoría surgió con el propósito de analizar los fenómenos traumáticos ocurridos durante la Segunda Guerra Mundial y por los procesos de descolonización subsecuentes. Asimismo, en el contexto latinoamericano la categoría de historia reciente se ha utilizado para comprender los sucesos ocurridos en las dictaduras $y$, mediante su enseñanza, se ha pretendido ejercer un contrapeso a las ideologías dominantes que se imponen tras alguna transformación social importante (Arias Gómez, 2015: 31-34).

Es pertinente subrayar que en la perspectiva de historia reciente hay algunas advertencias para el investigador: 1) existe el riesgo inminente de sesgar la información por la inmediatez de los sucesos analizados; 2) la vigencia en las interpretaciones puede acarrear dificultades de diversa índole, sobre todo, políticas; 3) la cercanía "hace que las pasiones e intereses que rodean a los mismos sigan vigentes y el historiador corre el riesgo de verse atrapado por esos impulsos" (López et al., 2010: 15); por lo anterior, se hace indispensable "la explicitación de las posiciones ético-políticas y morales" (Arias Gómez, 2015: 30).

En la revisión de literatura, efectivamente, se encontró que los trabajos que utilizan el concepto de historia reciente sí se encuentran vinculados con eventos sociales traumáticos; pero también se encontró un texto de Ángel Soto Gamboa (2004) titulado Historia del presente: estado de la cuestión y conceptualización, en el cual se consideran válidos en el constructo de historia del presente los términos coetánea, reciente, próxima o actual y no expone una diferenciación entre los conceptos reciente, o del presente. Sobre esta base, se consideró que el concepto de historia del presente colocaría el trabajo en una zona un poco más neutral. Cabe señalar que utilizar el concepto de historia del presente no exime al investigador de atender las advertencias señaladas anteriormente, ya que el concepto se encuentra en construcción (Soto Gamboa, 2004:102).

Para María Esther Aguirre Lora (2016) -investigadora del Instituto de Investigaciones sobre la
Universidad y la Educación (IISUE) de la Universidad Nacional Autónoma de México y asesora de esta investigación - el concepto de historia reciente debe revisarse con detenimiento. Considera que su complejidad radica en comprender la noción de cambio social implicada y también en comprender el proceso de desvelar "la necesidad de historia que tiene la sociedad para definir su futuro" (Aguirre Lora, 2016: 51). Al indagar sobre el concepto de cambio social, se encontró que existen cuatro o cinco grupos de teorías (Boudon, 1999: 302), entre ellas, un primer grupo dedicado a evidenciar las tendencias estructuradas sobre una base estadística. Para los propósitos de esta investigación el concepto de tendencias no se refiere a una cuestión estadística, sino a una situación de prácticas que son parte del sentido común de los miembros de una comunidad determinada. No se trata de encontrar tendencias en la mayoría de los programas en el país, sino de encontrar las prácticas que han sido tendencia en cada una de las instituciones que, si bien pueden ser contabilizadas e interpretadas por su frecuencia como una tendencia, también es posible que existan prácticas estadísticamente recurrentes poco significativas para la formación musical, como por ejemplo, la aplicación de exámenes departamentales de materias teóricomusicales, que no necesariamente marcarían una tendencia a la formación en composición.

De este modo, el cambio social - en el contexto de las jóvenes licenciaturas en música- tendría que interpretarse, más que por la frecuencia, por las prácticas que han modificado elementos curriculares sustantivos, como los perfiles de ingreso o egreso, o bien, en las que es posible reconocer cierto grado de estabilidad y aceptación por parte de una comunidad, aunque aún no sean susceptibles de análisis estadístico, como podrían ser los eventos extracurriculares que dependen del presupuesto disponible que, al no ser constante, podría interrumpir la frecuencia.

Pese a las divergencias sobre la conceptualización de esta temporalidad histórica, se dispone de 
definiciones que permiten establecer características comunes y retos metodológicos concretos. Para Julio Aróstegui, la historia del presente:

representa, por definición, análisis de procesos en curso o de aquellos que tiene alguna forma de vigencia inteligible en la vida actual. Frente a la historiografía tradicional que se caracteriza por el análisis de situaciones históricas de las que cabe decir que están concluidas, la dificultad aquí es, lógicamente, cómo captar y cómo analizar históricamente esas situaciones inacabadas" (Aróstegui, 2004: 44).

Asimismo, Ángel Soto Gamboa (2004: 106-107) define la historia del tiempo presente como "la posibilidad de análisis histórico de la realidad social vigente, que comporta una relación de coetaneidad entre la historia vivida y la escritura de esa misma historia, entre los actores y testigos de la historia y los propios historiadores".

Con el concepto de historia presente se pretende deslindar la implicación de sucesos traumáticos que, si bien pudieran haber ocurrido en el país, no están directamente relacionados con el interés central de las licenciaturas en cuestión. Esta precisión permite nuevamente alertar al investigador sobre la complejidad humana que, de acuerdo con Dilthey, no es en sí misma un impedimento para la generación de conocimiento, sino una característica intrínseca al mundo histórico: "la figuración del mundo histórico la acompañamos de amor y de odio, de apasionada alegría, de todo el ardor de nuestros afectos" (Dilthey, 1949: 45).

A diferencia de las ciencias de la naturaleza, que Dilthey (1949: 45-51) describió como serenas y mudas por ser externas, el mundo histórico está intervenido por una vida interior y un complejo juego de interacciones que dificultan la generación del conocimiento porque en él se hayan entrelazadas las unidades psíquicas de una cadena de generaciones en las que ya no es posible inferir directamente sus estados en tiempos anteriores; sin embargo, habrá que tener presente que la vida histórica desarrolla una articulación social de homogeneidad en los grupos; se generan formas permanentes y estables que se convierten en elementos de análisis social que pueden distinguirse claramente en los sistemas culturales: "La semejanza de los individuos es la condición para que se dé una 'comunidad"' (Dilthey, 1949: 47). Esta interacción entre los individuos, "conexión de fin" entre sus voluntades, no es resultado del azar, sino que es precisamente "por medio de esta interacción de los diversos individuos, de sus pasiones, de sus vanidades, de sus intereses [que] se verifica la trabazón necesaria de la historia de la humanidad" (Dilthey, 1949: 60).

Para los propósitos generales de este trabajo se delimitó el tratamiento heurístico al proceso de recopilación de documentos que evidenciaran todas aquellas acciones relacionadas con la conformación de las licenciaturas en música en el país alrededor de la década de 1990, las cuales pudieran ser interpretadas como parte de un sistema cultural con características estables. Las tendencias en las instituciones que se estudian en esta investigación son las que permiten bosquejar un futuro educativo determinado por la tradición, un concepto indispensable en las humanidades, el cual viabiliza el estudio de la continuidad, de la unidad y también de la creatividad (Ambrosio Velasco, en Ríos Ortega y Ramírez Velázquez, 2013: 4). Aquí se podría tomar en consideración la visión pragmática que destacó Carlos Pereyra al respecto de la tradición: "Una larga tradición encuentra el sentido de la investigación histórica en su capacidad para producir resultados que operen como guía para la acción" (Pereyra et al., 1980: 12).

De lo anterior se deduce que cada una de las licenciaturas en música en el país opera a partir de ciertas tradiciones y ha puesto en marcha acciones de formación específicas que la caracterizan como un sistema cultural que le permite diferenciarse de otros. Pese a las políticas educativas externas, que ejercen presión 
para el cambio de paradigma educativo, las prácticas se normalizan al interior de acuerdo con las valoraciones de la comunidad y se instituyen como tradición.

\section{La conciencia intencional de los actores y la interpretación}

Alfred Schütz ${ }^{3}$ y Harold Garfinkel ${ }^{4}$ desarrollaron una etnometodología con la que es posible explorar la conciencia intencional de los actores en el mundo de la vida (Lebenswelt) y conocer el significado atribuido a sus acciones. Esta conciencia intencional posee un sentido proyectivo y engloba razonamientos, conocimientos y valores compartidos que conforman el sentido común de una comunidad (De la Garza Toledo y Leyva, 2012: 217).

El sentido común se forma históricamente, es específico de cada sociedad y conforma el mundo de la vida que define el significado objetivo de las acciones. La finalidad de las ciencias sociales consiste precisamente en interpretar ese significado constituido desde el sentido común de la sociedad a la que pertenece el actor. (De la Garza Toledo y Leyva, 2012: 218).

Para Alfred Schütz, cada sociedad es poseedora de un acervo del conocimiento dentro del mundo de la vida, el cual debe comprenderse como una totalidad que no es completamente nítida, de ahí la necesidad de estudiar las zonas brumosas para comprender las significaciones que han otorgado a sus prácticas los miembros de una comunidad (Carbajal Vaca, 2014: 154).

Thomas Luckmann, discípulo y continuador de la fenomenología de Schütz subrayó que, dado que los mundos históricos humanos son producto de la acción social, éstos deben ser reconstruidos para comprender el sentido de las acciones que surgen en la relación entre cultura y estructura social. El acervo de significados que posee una cultura es el resultado de las formas organizadas de acción colectiva que se replican como formas reguladas por una estructura social que se ha institucionalizado en reglas y normas (Jochen Dreher, en De la Garza Toledo y Leyva, 2012: 124-125). Las normas de una comunidad no siempre han sido formalizadas ni materializadas en reglamentos. Las maneras de hacer en una sociedad se configuran y estabilizan en la práctica formando tradiciones que, en el caso de las instituciones educativas, pudieran haber trascendido las metas de los planes de estudio y la configuración curricular en general de las licenciaturas en música; de ahí la importancia de documentar la historia del presente a partir de las voces de los actores que han influido en las prácticas de cada comunidad académica; emprender esta tarea requiere herramientas etnográficas.

A partir de los postulados de Alfred Schütz, Harold Garfinkel (2006) acuñó el término etnometodología, el cual ha sido descrito por Pérez Hernáiz de la siguiente manera:

Por etno [Garfinkel] quería expresar, de alguna u otra manera, que los miembros de una sociedad tienen disponibles para su uso ciertos conocimientos que son del sentido común de esa sociedad, conocimientos sobre "cualquier cosa" [...] De modo que la etnometodología se refiere a un método que la gente posee. Es un conocimiento de los asuntos cotidianos que puede ser revelado en forma de razonamientos prácticos (Pérez Hernáiz, en Garfinkel, 2006: IX-X).

El tratamiento etnográfico, mediante instrumentos como la entrevista y la documentación de prácticas cotidianas a partir de la observación, permite explorar aquellas acciones concretas en el mundo de la vida que han sido institucionalizadas y han pasado a ser parte del acervo de conocimiento de una comunidad. Estas acciones, de carácter proyectivo, son las que dictan las tendencias en cada comunidad, por lo que las acciones deben ser interpretadas, cuidadosa y sistemáticamente, reconociendo las prácticas más estables y de mayor consenso.

3 (1899-1959) Sociólogo y filósofo austriaco que desarrolló el concepto mundo de la vida "Lebenswelt" de Edmund Husserl (1859-1938).

${ }^{4}$ (1917-2011) Sociólogo y fenomenólogo estadounidense. 
Para Dilthey (2000: 23) ser historiador es una manera de 'comprender' el mundo mediante lo singular para buscar una validez universal. Para ello es necesario "volver a sentir, comprender posteriormente al otro a través de una distancia temporal [que] es siempre comprensión de lo pasado, vuelta sobre él; es memoria, también memoria del otro". Esta comprensión singular, entendida como concientización de la propia individualidad, únicamente es posible en comparación con la de otros: "A este proceso por el cual conocemos un interior a partir de signos dados sensiblemente desde fuera lo llamamos: comprender" (ibid.: 25). Esta comprensión es semiótica y Dilthey la sostiene en lo siguiente: "todo lo que hacen los hombres puede ser interpretado como signo, expresión de una vida consciente; y sólo los signos, y nada más, pueden ser comprensibles y sometidos al comprender" (ibid.: 29).

El conocimiento de un sistema particular se lleva a cabo mediante un nexo de operaciones metódicas que se halla condicionado por el lugar que ocupa el sistema dentro de la realidad histórico-social. Sus medios son múltiples: análisis del sistema, comparación de las formaciones particulares que abarca, puesta en valor de las relaciones en que se halla este campo de la investigación con el conocimiento psicológico de las unidades de vida que constituyen los elementos de la interacción que forma el sistema y, por otra parte, con el contexto histórico-social de donde ha sido destacado a los fines de la investigación. Pero el proceso cognoscitivo es uno solo (Dilthey, 1949: 65).

La hermenéutica permite estudiar la comunicación que ha existido entre los actores de las IES en relación con su realidad histórico-social; misma que debe ser fijada en signos lingüísticos: "sólo en el lenguaje encuentra lo interior humano una expresión que sea completa, exhaustiva y objetivamente comprensible. De ahí que el arte de comprender tenga su centro en la exégesis o interpretación de los vestigios de existencia humana contenidos en la escritura" (Dilthey, 2000: 31); para lograr tal interpretación - fin primordial de la hermenéutica - es necesario recopilar lo documentado y documentar las voces de los actores contemporáneos para encontrar coincidencias y desacuerdos.

La hermenéutica propuesta por Dilthey (2000: 13) incorpora la volición y los afectos que son parte del ser humano; una visión distinta a la cientificista centrada sólo en la cognición. Desde esta óptica, se infiere que las políticas públicas que han transformado las prácticas educativas en las últimas tres décadas han estado intervenidas por las voluntades y las preferencias de los actores en turno, de ahí que cada programa disponga de características particulares, no necesariamente coincidentes con otros en el país.

En otras palabras, aunque en apariencia las IES y los programas de licenciatura en música comparten significaciones, la formación de cada comunidad es un proceso único. Cada sistema cultural ha sido influido por las concepciones de sus actores y sus interacciones en un entramado de coincidencias, desacuerdos y resistencias. Estas influencias pueden haberse fijado, más que en los programas de estudio, en prácticas académicas y de convivencia que se propagan en un currículo oculto.

Desde la perspectiva de la pedagogía crítica de Giroux, la educación es una práctica política, social y cultural que debe ser analizada para comprenderla (Carbajal Vaca, 2015: 445; 2016b: 45-47). El dominio neoliberal de los últimos 30 años en México ha propiciado un discurso educativo enfocado en el desarrollo de competencias laborales alcanzadas mediante modelos instruccionales. Giroux (2004: 143-147) dispone en su teoría de la resistencia cuatro categorías que conforman un constructo teórico para analizar la acción colectiva generada en las relaciones de poder entre escuela y sociedad que pudiera ayudar a comprender la configuración del currículo oculto. Estas cuatro categorías son: la intencionalidad, la conciencia, el significado del sentido común, y la naturaleza y valor del comportamiento no discursivo. Desde esta perspectiva, cada programa de licenciatura es reflejo de las intencionalidades 
de los sujetos involucrados en el diseño curricular, pero también en las prácticas no formalizadas o institucionalizadas curricularmente. Se asume que los miembros de una comunidad tienen cierto grado de conciencia sobre sus propias prácticas; sin embargo los actores podrían ser también reproductores de prácticas que forman parte del sentido común de la institución; prácticas que se replican y que, por ser una tradición afianzada, ya no se cuestionan. Asimismo, observar y analizar lo que se hace y no lo que se dice o se escribe, podría ser una manera de registrar el comportamiento no discursivo de cada entorno. Es en este sentido que emerge la noción de currículo oculto, el cual es descrito por Giroux (2004: 72-74) como "normas, creencias y valores no declarados, implantados y transmitidos a los alumnos por medio de reglas subyacentes que estructuran las rutinas y las relaciones sociales en la escuela y en la vida en las aulas", el cual tiene la función de conservar la sociedad existente. En el caso de los programas de licenciatura, se entiende que en las prácticas se podrán percibir tendencias distintas: a mantener la tradición del conservatorio que precedió a los modelos universitarios; a cultivar prácticas musicales contemporáneas, académicas o populares; a implementar prácticas educativas inclusivas; a impulsar la investigación y divulgación de conocimiento; entre otras.

\section{Algunos resultados}

Uno de los retos más grandes de este proyecto ha sido la vinculación con académicos en las entidades federativas que disponen de programas de licenciatura. Aunque el proyecto inició formalmente en enero de 2017, desde 2016 se comenzó a gestionar la participación de colaboradores de distintas instituciones en el país.

En 2016 se presentó el proyecto en la mesa redonda Situación de la Educación Musical en México, en el Encuentro Internacional de Educación Musical en la Benemérita Universidad Autónoma de Puebla (BUAP). En diciembre se solicitó a los participantes la firma de una carta de consentimiento informado y se recibió respuesta de trece investigadores de las siguientes instituciones: Universidad Autónoma de Aguascalientes (UAA), Universidad Autónoma de Zacatecas, Universidad de Guadalajara, Universidad de Colima, Universidad Autónoma de Nayarit, Universidad Autónoma de Chihuahua, Universidad de Guanajuato, Escuela de Música del Estado de Hidalgo y Universidad Autónoma del Estado de Hidalgo. En el primer simposio de colaboradores, realizado en junio de 2017 en el marco del Coloquio Internacional de Educación Musical a Nivel Superior (CIEMNS), se socializaron los primeros hallazgos de la Universidad Autónoma de Aguascalientes, la Universidad de Colima, la Universidad Autónoma de Nayarit, la Universidad Autónoma de Chihuahua y el Colegio de San Juan Siglo XXI, Tamaulipas, documentado por un investigador de la Universidad Autónoma de Aguascalientes.

Este encuentro fue de vital importancia porque se tuvo la oportunidad de conocer una primera percepción de los colaboradores sobre las actividades propuestas. En julio y agosto se esbozó el marco teórico-metodológico y el 26 de septiembre de 2017 fue socializado por uno de los colaboradores en el $9^{\circ}$ Simposio de Música "La Música en Latinoamérica", durante la $2^{\text {a }}$ Semana Internacional de las Artes en el Instituto de Artes de la Universidad Autónoma del Estado de Hidalgo. Un mes más tarde, el 26 de octubre de 2017, se presentó la propuesta a distancia desde Alemania en la mesa de trabajo La educación musical y sus problemáticas, realizada durante el $4^{\circ}$ Encuentro Internacional de Educación Musical en la BUAP. En noviembre de 2017 se presentó la ponencia Historia reciente de la educación musical de nivel superior en México: un acercamiento a los retos curriculares de la licenciatura en música de la Universidad Autónoma de Aguascalientes, en el XIV Congreso Nacional de Investigación Educativa (COMIE), en San Luis Potosí, y se expusieron tres de los retos curriculares que ha enfrentado la licenciatura en Música de esta Universidad: 1) formar músicos en el contexto universitario, 2) comprender el 
currículo departamental, 3) investigar, difundir, vincular e internacionalizar (Carbajal Vaca et al., 2017). En diciembre de 2017 se diseñó una guía de entrevista a informantes, la cual aplicaron algunos de los colaboradores. Este proyecto también ha permitido integrar trabajos que los estudiantes desarrollan en sus asignaturas; por ejemplo, el de la estudiante Olga Servín Nájera, que estudió los factores que influyeron en la elección de carrera de los estudiantes de la licenciatura en Música de la UAA. Los resultados fueron presentados el 13 de septiembre de 2018 en el Seminario Permanente (SEMPER) del Departamento de Música de la UAA.

El 29 de junio de 2018, en el marco del CIEMNS, se realizó el segundo simposio de colaboradores. Participaron nueve de los colaboradores registrados, entre ellos María Esther Aguirre Lora, ${ }^{5}$ quien ha sido asesora externa del proyecto y cuya intervención ha sido determinante en la comprensión de la perspectiva histórica de este trabajo. El análisis de Aguirre Lora (2018), compartido con los colaboradores durante el segundo simposio en junio de 2018, evidenció los retos y los alcances del estudio. La investigadora enfatizó la necesidad de observar los distintos movimientos ocurridos a lo largo de los siglos XX y XXI, que nos han posicionado necesariamente en una perspectiva multidisciplinaria que es muy distinta a la monodisciplinaria y lineal a la que se recurría anteriormente en los estudios históricos. Para esta autora es importante que se observe la transformación en la perspectiva histórica, que ha ido desde la historia idealista, pasando por la historicista, la positivista, la social y la historia cultural, hasta llegar a la historia social de lo cultural, la cual focaliza el interés en el proceso y no en el hecho mismo; una perspectiva que fue conveniente para este trabajo, ya que en esta óptica es posible interpretar y entender las paradojas y los dilemas que se dan en la invención de las tradiciones y las prácticas que se viven en las instituciones.
Esta comprensión es lo que hace posible la conservación de la memoria y del patrimonio educativo; de ahí que el rescate de partituras, de instrumentos, de documentos y de métodos puedan ser percibidos como el rescate del patrimonio cultural. La investigadora también alertó al equipo de trabajo sobre el peligro que existe en virar el interés de investigación constantemente, porque el trabajo podría perfilarse a conocer los sujetos, los actores, los agentes y a comprender cómo las intervenciones de los sujetos atraviesan el currículo y lo construyen desde su propia trayectoria y no sólo como un ente en abstracto, desarticulado de la realidad. Asimismo, se podría enfocar en conocer las comunidades y sus procesos de socialización de la música y la educación musical, lo que derivaría en conocer de manera profunda lo que ha ocurrido en las instituciones, con sus sistemas, sus políticas, sus lenguajes y las revoluciones tecnológicas. Otra perspectiva podría ser conocer qué ha ocurrido con el mundo de la cultura y tradición oral que se diluye en un mundo dominante de cultura escrita. También podrían estar implicadas otras dimensiones importantes, como la geográfica, la de la subjetividad, la infancia, la juventud, la adultez, el género, los grupos étnicos, los grupos migrantes, la marginación, la corporeidad, entre otros aspectos, que darían un giro al campo de investigación. Se podría dirigir la mirada hacia las comunidades sociales, las académicas, las culturales, las asociaciones, los grupos de investigación, las redes y los procesos de socialización, lo cual también generaría conocimiento sobre la conformación de los programas. Señaló que un campo muy rico es la historia intelectual, con la que se construyen biografias que dejan entrever trayectorias, legados, las propuestas formativas y sus procesos de apropiación. Asimismo, sugirió que cada uno de estos subcampos o líneas son muy importantes en la construcción de estados de conocimiento.

${ }^{5}$ Profesora en el Posgrado en Pedagogía, en el Posgrado en Educación Musical de la Facultad de Música e investigadora de carrera en el IISUE, UNAM. 
A manera de guía, recomendó que se tomara en consideración que en las instituciones se realizan foros nacionales e internacionales, reuniones, se desarrollan investigaciones, se publica, se dialoga, y todo esto conforma una gran fuente de información en la que se encuentran indicadores del desarrollo y de las tendencias en una institución. También existe otro tipo de fuentes que podrían ser exploradas, como los archivos, los manuales, los documentos curriculares, las escenografías, las fuentes orales, las iconográficas, las sonoras, entre otras, las cuales podrían ser exploradas desde la perspectiva semiótica, o bien, desde la historia comparada. En cualquier caso, Aguirre Lora enfatizó que los colaboradores habrían de comprometerse a:

pensar históricamente los problemas del campo de la educación musical, y esto quiere decir: que la historia es como una clave para la producción de conocimiento, es una clave muy importante, clave epistémica y si yo no hago historia de la educación [entonces] tengo obligación - entre comillas - de enfocar desde la historia, entender desde la historia, problematizarme desde ahí. Porque las cosas no aparecen de la noche a la mañana, sino que hay una configuración. Se persisten prácticas, se traslapan prácticas, se actualizan prácticas y, en ese sentido, la historia es una forma de entender, de inteligir [...] el fin histórico del presente (Aguirre Lora, 2018).

Entre las fuentes de información que podrían ser exploradas, Aguirre Lora llamó la atención hacia la de las escenografías. Juan Pablo Correa, de la UAA, trajo a la mesa de diálogo la reflexión sobre el papel que juegan en esta comprensión los edificios donde están ubicadas las instituciones. En principio, porque en varias universidades el Departamento o Facultad de Música se encuentra en un edificio alejado de los campus universitarios, lo que podría esconder intencionalidades no declaradas formalmente.

Aguirre Lora comentó que los edificios son un tema de especial riqueza en el terreno de las artes, que quizá no ha sido suficientemente explorado. Enfatizó que habría que tomar en cuenta que en el momento en que uno problematiza un elemento, se encuentra ya en el terreno de la investigación, por lo que un proyecto podría encargarse perfectamente de estudiar la manera como se dispone de los espacios, por ejemplo: la manera como se sienta el auditorio en las presentaciones; si existe un estrado o se ubican sentados dentro de un grupo. ${ }^{6}$ También sería posible estudiar el vestuario, la organización de los músicos, entre otros aspectos proveedores de información sobre las comunidades.

Sobre esta misma temática de las edificaciones surgieron otros comentarios, como el de Mayra Analía Patiño Orozco, de la Universidad de Colima, quien ya había comenzado un diálogo con otros colegas de su universidad sobre el significado de los edificios donde se ubican los departamentos y facultades de música que, en casos como el de la Universidad de Guadalajara, o la Universidad de Guanajuato, son edificios históricos. Así, Patiño Orozco relató que el edificio que ocupaba la Escuela de Música en Colima, en el Instituto de Bellas Artes (IUBA), fue la primera sede de la Universidad de Colima y también donde se tuvieron las primeras facultades de Medicina y Contabilidad. Es interesante señalar que este edificio, antes de ser sede de la Universidad, fue una hacienda y después un hospital. A colación de este comentario, Carbajal Vaca expresó una inquietud sobre la posibilidad de asociar a la perspectiva de historia reciente algunos acontecimientos importantes, como el terremoto de 2003 que causó la destrucción del edificio del IUBA; o la repentina e inexplicable demolición de la finca donde se encontraba la Escuela de Música de la Universidad de Guadalajara en la década de 1980 (ver Nuño, 2016).

${ }^{6}$ Como lo hizo Aguirre en el Simposio, ella se sentó en el centro y se rodeó de los colaboradores y participantes, de una manera muy amigable y amena. 
Aguirre Lora precisó que la concepción de trauma a la que aluden los teóricos de historia reciente es la de trauma social brutal, por ejemplo, el causado por la Segunda Guerra Mundial, el exilio argentino o el exilio español; de ahí el interés por saber cómo lo vivieron quienes lo sufrieron. Subrayó que en este proyecto de investigación la connotación más importante del término historia reciente está relacionada con saber qué está ocurriendo hoy en día con las licenciaturas en música; es decir, en el presente:

los historiadores están trabajando con el presente. Desde el campo que estén trabajando, como quiera que estén trabajando pero que están viviendo aquí. Sus preguntas surgen de este presente. Y sobre eso se va haciendo el pasado. Yo creo que sí hay una preocupación por rescatar desde el presente qué es lo que ha pasado con las licenciaturas en música, con todos sus traumas. Cada quien tiene sus traumas (Aguirre Lora, 2018).

Ante la exposición de Aguirre Lora, fueron surgiendo otras reflexiones que apuntan hacia otro tipo de investigaciones posibles, como el hecho de que fuera Griselda Álvarez, primera gobernadora mujer en México, la principal impulsora de la profesionalización de lo que en algún tiempo fueron los talleres de Artes y Oficios en Colima y de que se haya logrado la sistematización de la enseñanza de las artes mediante un acuerdo con el rector Humberto Silva para la creación del Instituto Nacional de Bellas Artes.

Por su parte, Adriana Martínez Maldonado comentó el caso de la Universidad de Guanajuato, ubicando el análisis en la partida de los jesuitas. Cuando fueron expulsados de la América novohispana, habían formado una escuela de Artes y Oficios, en un edificio adjunto a lo que es la compañía de Jesús. La Escuela de Artes y Oficios se relaciona a principios del siglo XX con la política de modernidad de
Porfirio Diaz, que trae la zarzuela, las bandas, enriquece otro contexto donde se crea el Teatro Juárez.

Los comentarios de los colaboradores evidencian las distintas ópticas desde las que cada miembro de una comunidad vivencia su entorno; problemática advertida por Aguirre Lora. Este simposio ofreció la posibilidad de exponer la diversidad temática, pero al mismo tiempo fue una oportunidad para puntualizar los propósitos del proyecto, sobre todo, para los colaboradores que se integraron posteriormente, como fue el caso de Guillermo Vargas Rodríguez, de la Universidad Autónoma de Querétaro (UAQ), quien expuso las dificultades que ha enfrentado para lograr las entrevistas a los informantes. En esta colaboración se perfilaron las primeras apreciaciones que podrían dar pauta a la categoría de tendencias; se apreció que el marco teórico que se puso a disposición comenzó a intervenir en las comprensiones, por ejemplo, cuando Vargas Rodríguez ubicó las licenciaturas de la UAQ como programas que experimentan un proceso de transición de una perspectiva excluyente hacia una más incluyente.

\section{Reflexiones}

El logro más sólido hasta el momento es la definición de una herramienta teórico-metodológica para documentar y analizar los programas desde el enfoque de la historia del presente, la cual podrá ser utilizada para dar seguimiento tanto a los resultados de esta investigación, como para iniciar la documentación de otros programas ausentes en este primer acercamiento.

Los dos simposios del proyecto han permitido el acercamiento entre colegas que compartimos inquietudes similares. La red de colaboración que se ha tejido ha ido ampliando paulatinamente la gama de actividades que nutren la formación de los estudiantes del Departamento de Música de la UAA y ha fortalecido los vínculos académicos interinstitucionales.

Entendemos que la documentación histórica, en el marco de eventos traumáticos de gran magnitud - característica de la perspectiva de historia reciente-, 
la realizan de manera sistemática los periodistas, los activistas sociales y los investigadores. Cuando las perspectivas se entrecruzan, se dialogan y se discuten en la prensa, revistas, documentales y libros, se tiene la posibilidad de proponer hipótesis para analizar causas, efectos y otros factores de interés para la investigación. En cambio, investigar procesos socioculturales relativamente pacíficos, como la educación musical de nivel superior, que no están en el interés profundo de los periodistas ni de la opinión pública, es una tarea compleja porque la información documentada es escasa y escueta.

La ausencia de eventos traumáticos de alcance masivo sitúa esta investigación en un espacio alejado de la perspectiva de historia reciente; sin embargo, aquí habría que subrayar - como lo destacaron algunos de los colaboradores en los simposios realizados en
2017 y 2018 - que sí han existido eventos con implicaciones políticas destacables, que han marcado las prácticas educativas y que, aunque no podría compararse con la magnitud de una guerra, sí han provocado cambios en la demanda profesional y las prácticas musicales, situaciones que podrían ser consideradas en la categoría de cambio social.

En síntesis, la perspectiva de la historia del presente focaliza la actividad de documentación como una responsabilidad universitaria y de sus jóvenes cuerpos académicos. Disponer de las primeras publicaciones, aunque los resultados sean modestos, permite abrir el diálogo y despertar el interés de otros investigadores. Con este trabajo se está cumpliendo la meta de documentar las voces del presente, que quedarán a disposición para ser analizadas y contrastadas con voces futuras. 


\section{Referencias}

Aguirre Lora, M. E. (2018), "Conferencia", presentada en el Segundo Simposio de Colaboradores del proyecto PIE 171. Historia reciente de la educación musical de nivel superior en México, Aguascalientes, Universidad Autónoma de Aguascalientes, 29 de junio.

Aguirre Lora, M. E. (2016), Historia e historiografia de la educación en México: hacia un balance, 2002-2011. Vol. I y II, México, ANUIES/Consejo Mexicano de Investigación Educativa (Colección Estados del Conocimiento).

Aguirre Lora, M. E. (2006), "La Escuela Nacional de Música de la UNAM (1929-1940). Compartir un proyecto", Perfiles Educativos, 28(111), pp. 89-111, <http://www.scielo.org.mx/pdf/peredu/v28n 111 / n11 la5.pdf> [Consulta: octubre de 2019].

Alonso, L. (2007), "Sobre la existencia de la historia reciente como disciplina académica. Reflexiones en torno a historia reciente", en M. Franco y F. Levín, (comps.), Perspectivas y desafios de un campo en construcción, Rosario, Consejo Nacional de Investigaciones Científicas y Técnicas, pp. 191-201.

Arias Gómez, D. H. (2015), "La enseñanza de la historia reciente y la formación moral. Dilemas de un vínculo imprescindible", Folios (Segunda época), núm. 42, pp. 29-41, <http://www.scielo.org.co/pdf/folios/n42/ n42a03.pdf> [Consulta: agosto de 2018].

Aróstegui, J. (2004), "La historia del presente. ¿Una cuestión de método?", en C. Navajas Zubeldia (ed.), Actas de IV Simposio de Historia Actual, Logroño, Gobierno de La Rioja/Instituto de Estudios Riojanos, 17-19 de octubre de 2002, pp. 41-75.

Beuchot Puente, M. (1999), Heurística y hermenéutica, México, Centro de Investigaciones Interdisciplinarias en Ciencias y Humanidades, UNAM.

Boudon, R. (1999), "Las teorías del cambio social", Polis. Investigación y análisis sociopolitico y psicosocial, <https:// revistas-colaboracion.juridicas.unam.mx/index.php/ polis/article/view/16734/14968> [Consulta: enero de 2018].

Carbajal Vaca, I. S. (2016a), "De músico a investigador histórico: una ruta sinuosa", en C. Lorenzo Monterrubio (coord.), Perspectiva Histórica del Arte I, Pachuca de Soto, Universidad Autónoma del Estado de Hidalgo, pp. 141-162, <https://repository.uaeh.edu.mx/bitstream/ bitstream/handle/123456789/17329/perspectiva_ historica_del_arte_1.pdf?sequence $=1 \&$ is Allowed $=\mathrm{y}>$ [Consulta: septiembre de 2019]

Carbajal Vaca, I. S. (2016b), "Del modelo de conservatorio al modelo universitario: la experiencia de transición en el Departamento de Música de la Universidad de Guadalajara", en A. Barrera Aguilar y D. Pérez Navarro (comps.), Modelos educativos: ¿Cómo ir en otra dirección? (Vol. II. Tomo I. Modelos Educativos Alternativos, Libro Electrónico), Tepic, Universidad Autónoma de Nayarit, pp. 40-52, <https://media.wix.com/ ugd/4beb15_e2cf0b1cfb5743b6b52e8180bede73c5. pdf $>$ [Consulta: abril de 2017].

Carbajal Vaca,I. S. (2015), “CConservatorios Universitarios? Evaluación de resistencias en los programas de educación musical de nivel superior", ponencia presentada en el $11^{\circ}$ Congreso de Investigación Educativa Internacional, 21-24 de octubre, Nuevo Vallarta, Universidad Autónoma de Nayarit.

Carbajal-Vaca, I. S. (2014), Acercamiento semiótico y epistemológico al aprendizaje de la música, Guadalajara, Jalisco, Universidad de Guadalajara (Colección Graduados. Serie Sociales y Humanidades 6), <http://www.publicaciones.cucsh.udg.mx/pperiod/ cgraduados/pdf/2012/acercamiento2012.pdf > [Consulta: enero de 2019].

Carbajal Vaca, I. S., J. P. Correa Ortega y R. W. Capistrán Gracia (2017), "Historia reciente de la educación musical de nivel superior en México. Un acercamiento a los retos curriculares de la licenciatura en Música de la Universidad Autónoma de Aguascalientes", en Memoria Electrónica XIV Congreso Nacional de Investigación Educativa, 3(3) 2017-2018, San Luis Potosí, COMIE <http:// www.comie.org.mx/congreso/memoriaelectronica/ v14/doc/0351.pdf> [Consulta: abril de 2017]. 
Conservatorio Nacional de Música (CNM) (2017), Conservatorio Nacional de Música. Objetivo de la licenciatura, México, INBA, <http://www.conservatorio.bellasartes. gob.mx/oferta-educativa.html> [Consulta: abril de 2017].

De la Garza Toledo, E. y G. Leyva (2012), Tratado de metodología de las ciencias sociales: perspectivas actuales, México, Fondo de Cultura Económica.

Dilthey, W. (1949), Obras de Wilhelm Dilthey. Introducción a las ciencias del espiritu (Original en alemán 1883), México, Fondo de Cultura Económica.

Dilthey, W. (2000), Dos escritos sobre hermenéutica. El surgimiento de la hermenéutica y los Esbozos para una crítica de la razón histórica, Madrid, Istmo.

Franco, M. y F. Levín (2007), "El pasado cercano en clave historiográfica", en M. Franco y F. Levín (comps.), Perspectivas y desafios para un campo en construcción, Buenos Aires, Paidós, <http://files.pluriversidad-oikologias. webnode.es/200000019-2c6502d5e4/2013\%20 CA PACITACI O N \% 20 PR R E N G I A L \% 20 FRANCO \%20LEVIN.pdf> [Consulta: junio de 2017].

Gaos, J. (1960), "Notas sobre la historiografía", en Historia Mexicana, 9(4), (s.l.), pp. 481-508. <http:// historiamexicana.colmex.mx/index.php/RHM/ article/view/885/776> [Consulta: mayo de 2017].
Garfinkel, H. (2006), Estudios en etnometodología, Barcelona, Anthropos/UNAM.

Giroux, H. (2004), Teoría y resistencia en educación. Una pedagogía para la oposición, México, Siglo XXI.

López, M., G. Figueroa y B. Rajland (eds.) (2010), Temas y procesos de la historia reciente de América Latina, Santiago de Chile, CLACSO/Arcis <http://biblioteca.clacso.edu. ar/clacso/coediciones/20100827084236/historiarec. pdf> [Consulta: junio de 2017].

Matute, A. (1999), Heurística e Historia, México, UNAM.

Nuño, A. S. (2016), "Revelan que Flavio avaló destrucción", El Diario NTR, <http://www.ntrguadalajara.com/post. php?id_nota $=53001>$ [Consulta: febrero de 2019].

Pereyra, C., L. Villoro, L. Gonzáléz, J. J. Blanco, E. Florescano, A. Córdova, H. Aguilar Camín, C. Monsiváis, A. Gilly y G. Bonfil Batalla (1980), Historia, ¿para qué?, México, Siglo XXI.

Ríos Ortega, J. y C. A. Ramírez Velázquez (2013), Agendas de investigación en bibliotecología e información: tendencias nacionales e internacionales, México, UNAM.

Soto Gamboa, A. (2004), "Historia del presente: estado de la cuestión y conceptualización", Historia Actual Online, núm. 3, pp. 101-116, <https://www.historiaactual.org/Publicaciones/index.php/hao/article/ view/34> [Consulta: febrero de 2019]. 